\title{
Rhodnius pallescens (Barber, 1932) y Trypanosoma cruzi en Urabá
}

\author{
Elisa Londoño, Diana M. Isaza
}

\section{Resumen}

Se informa por primera vez para la zona de Urabá (Antioquia), la presencia de Rhodnius pallescens infectado con Trypanosoma cruzi. El ejemplar era una hembra que fue identificada con las claves de Lent y Wigodzinsky y confirmada en el Instituto Nacional de Salud. De ella se obtuvieron huevos fértiles que fueron usados para establecer una colonia en el Instituto Colombiano de Medicina Tropical; presentaba, además, heces positivas para T. cruzi, parásito que fue identificado bioquímicamente en el Centro Internacional de Entrenamiento e Investigaciones Médicas (CIDEIM, Cali).

\section{Rhodnius pallescens (Barber, 1932) and Trypanosoma cruzi in Urabá, Antioquia Department, Colombia}

We report the first finding of Rhodnius pallescens infected with Trypanosoma cruzi in the Urabá region (Antioquia). The insect was a female identified with the keys of Lent and Wigodzinsky. It was confirmed by the Instituto Nacional de Salud in Colombia. Fertile eggs were obtained and the new born insects were used to establish a colony of R. pallescens in the Instituto Colombiano de Medicina Tropical in Medellín. The faeces were found positive for Trypanosoma cruzi. The parasite underwent biochemical identification in the Centro Internacional de Entrenamiento e Investigaciones Médicas (CIDEIM, Cali).

La enfermedad de Chagas es producida por el parásito Trypanosoma cruzi, presente en las heces de insectos hematófagos de la subfamilia Triatominae, conocidos en Colombia con el nombre común de "pitos".

Rhodnius prolixus es el insecto vector más importante de $T$. cruzi y $T$. rangeli en Colombia, encontrándose de manera abundante en las principales regiones endémicas para la enfermedad de Chagas: región del Catatumbo, región oriental y valle del río Magdalena (1). En el país se han informado otros triatomíneos infectados, con menor importancia como vectores del parásito; entre ellos, Rhodnius pallescens, el cual se ha encontrado en la costa atlántica infectado con $T$. cruzi y $T$. rangeli $(1,2)$ y en el Magdalena medio, colonizando el domicilio humano e infectado con T. cruzi (3).

Este insecto es el principal vector de T. cruzi y $T$. rangeli en Panamá; es, además, el triatomíneo más común en habitaciones humanas (1). En el centro del país, se ha demostrado que tiene preferencia alimenticia por el hombre (4).

El presente artículo informa por primera vez $R$. pallescens infectado con $T$. cruzi para la zona de Urabá. El hallazgo se realizó dentro de una casa, en la vereda Aguas Frías del municipio de Turbo (Antioquia). Se encontró una hembra viva 
que fue trasladada a los laboratorios del Instituto Colombiano de Medicina Tropical en Medellín donde hizo posturas de huevos fértiles que fueron usados para establecer una colonia. Este ejemplar fue identificado con las claves de Lent y Wygodzinsky (5) y confirmado por el Grupo de Entomología del Instituto Nacional de Salud. Sus heces estaban positivas para flagelados, los cuales fueron aislados en ratones, cultivados en medio de Novy-Nicolle-McNeal (NNN) (6) y, luego, identificados bioquímicamente en el CIDEIM como T. cruzi.

Aunque el departamento de Antioquia no es considerado como región endémica para la enfermedad de Chagas, presenta triatomíneos de diferentes especies hacia la zona de Dabeiba y el Magdalena medio $(3,7)$. El presente registro amplía la distribución conocida de $R$. pallescens y $T$. cruzi en Antioquia y en el país y alerta sobre la posibilidad de que el parásito se esté estableciendo en la zona, dadas sus condiciones ambientales óptimas para albergar todos los elementos del ciclo.

\section{Agradecimientos}

Al doctor Alberto Morales del Grupo de Entomología del Instituto Nacional de Salud por la confirmación de la identificación de $R$. pallescens.
Al Centro Internacional de Entrenamiento e Investigaciones Médicas, CIDEIM, por la caracterización bioquímica de la cepa de $T$. cruzi.

\section{Referencias}

1. D'Alessandro A, Barreto P, Duarte RCA. Distribution of triatomine-transmitted trypanosomiasis in Colombia and new records of the bugs and infections. J Med Ent 1971;8:159-72.

2. Marinkelle JC. Colombian triatominae and their infestation with trypanosomatid flagellated. Mitt Inst Col Al Invest Cient 1972;6:13-29.

3. Moreno J. Recientes estudios epidemiológicos de tripanosomiasis americana en diferentes áreas de Colombia. Biomédica 1991;11(Supl.1):43-4.

4. WHO. Control of Chagas disease. Technical Report Series $811 ; 1991: 19$

5. Lent $\mathbf{H}$, Wygodzinsky P. Revision of the triatominae (Hemiptera, Reduviidae) and their significance as vectors of Chagas disease. Bulletin of the American Museum of Natural History 1979;163 (Art.3):416-8.

6. Botero D, Restrepo M. Técnicas de laboratorio en parasitología médica. En: Parasitosis humanas. Segunda edición. Medellín: CIB; 1992:402.

7. Restrepo M, Morales A, Ferro C. Presencia de Triatoma dispar Lent, 1950 en Colombia (Hemiptera, Reduviidae). Boletín Epidemiológico de Antioquia 1989; XIV(1-2):109. 